\title{
Closed-form solutions for accelerated MHD flow of a generalized Burgers? fluid in a rotating frame and porous medium
}

\author{
Ilyas Khan ${ }^{*}$, Farhad Ali $^{2}$, Norzieha Mustapha ${ }^{3}$ and Sharidan Shafie ${ }^{4}$
}

\section{"Correspondence:}

ilyaskhanqau@yahoo.com

'Department of Basic Sciences,

College of Engineering, Majmaah

University, P.O. Box 66, Majmaah,

11952, Saudi Arabia

Full list of author information is

available at the end of the article

\begin{abstract}
Closed-form solutions for magnetohydrodynamic (MHD) and rotating flow of generalized Burgers? fluid past an accelerated plate embedded in a porous medium are obtained using the Laplace transform technique. Modified Darcy?s law for generalized Burgers? fluid is taken into account. Both constant and variable acceleration cases are considered. The graphical results along with illustrations are presented to bring out the effects of indispensable parameters on the velocity. The obtained solutions are reduced as special cases to their limiting solutions by taking some suitable parameters equal to zero.
\end{abstract}

Keywords: accelerated flow; generalized Burgers? fluid; MHD; porous medium; exact solutions

\section{Introduction}

Most of the studies dealing with flow phenomena of rotating fluids in porous media are limited merely to Newtonian fluids. This is because of the fact that the governing equations of Newtonian fluids are simpler in comparison to non-Newtonian fluids. Non-Newtonian fluids, on the other hand, recently have become quite prevalent in industry and engineering. Some of their common examples are butter, cosmetics and toiletries, mud, jams, jellies, blood, certain oils like waxy crude oils, palm oil, coconut oil, shampoo and soap. As a consequence of diverse physical structures of these fluids, there is not even a single constitutive model which can predict all the salient features of non-Newtonian fluids. Generally, there are three non-Newtonian fluid models. They are known as (i) the differential type, (ii) the rate type, and (iii) the integral type. But the most famous amongst them are the first two models. In this article, we study the second model, the rate type fluid, and consider its subclass known as generalized Burgers? fluid. The simplest subclasses of rate type fluid are Maxwell and Oldroyd-B fluids. But they are limited in terms of their applications. The Burgers? fluid model is capable of the description of motion of the earth?s mantle, response of asphalt concrete, geological structures modeling, for instance, Olivine rocks, and the propagation of seismic waves in the interior of the earth $[1,2]$. On the other hand, the analysis of the effect of rotation of electrically conducting fluids plays an important role in cosmical fluid dynamics, energy generators, structure of rotating magnetic stars, magneto-geophysics and planetary astrophysics especially in the study of wind generated

(c) 2015 Khan et al.; licensee Springer. This is an Open Access article distributed under the terms of the Creative Commons Attribution License (http://creativecommons.org/licenses/by/4.0), which permits unrestricted use, distribution, and reproduction in any medium, provided the original work is properly credited. 
currents on rotating earth. The solutions for rotating flow of non-Newtonian fluids become more difficult when these fluids are considered in the presence of MHD and porous medium. Despite these difficulties, various researchers are involved in the field and are making their interesting contributions. However, the solutions to such problems are very rare, specially if one is interested in the closed form solutions. Some interesting contributions in this direction are mentioned in references [3-21]. A few other investigations of non-Newtonian fluids consider the effect of rotation are reported by Shahzad et al. [15], Hayat et al. [16-19], Siddiqui et al. [20] and Abelman et al. [21]. Recently, Khan et al. [22] obtained the exact solutions for accelerated flows of a rotating second grade fluid in a porous medium using the Laplace transform technique.

Based on this motivation, the present article studies the unsteady MHD flow of generalized Burgers? fluid with modified Darcy?s law. Two explicit examples of acceleration subject to a rigid plate are considered. The closed form solutions of the resulting problem are developed by using the Laplace transform technique. The velocity profiles are plotted in order to illustrate the variation of embedded flow parameters.

\section{Flow due to a constantly accelerated plate}

Let us consider an incompressible generalized Burgers? fluid bounded by a rigid plate at $z=0$. The fluid occupies the porous half space $z>0$. The $z$-axis is taken normal to the plate. Initially, the plate and fluid are at rest. After time $t=0^{+}$, both the fluid and the plate perform solid body rotation with uniform angular velocity $\boldsymbol{\Omega}$ about the $z$-axis. The fluid is electrically conducting under the action of a uniform magnetic field $\mathbf{B}=\mathbf{B}_{0}+\mathbf{b}$, acting parallel to $z$-axis. Here $\mathbf{B}_{0}$ is an applied magnetic field and $\mathbf{b}$ is an induced magnetic field. The electric field is assumed to be zero. The magnetic Reynolds number is taken small to neglect the effect of induced magnetic field. The flow in the fluid is induced due to the constantly accelerating plate. The governing flow equation is given by

$$
\left(1+\lambda_{1} \frac{\partial}{\partial t}+\lambda_{2} \frac{\partial^{2}}{\partial t^{2}}\right)\left(\frac{\partial F}{\partial t}+2 i \Omega F\right)=\left[\begin{array}{c}
v\left(1+\lambda_{3} \frac{\partial}{\partial t}+\lambda_{4} \frac{\partial^{2}}{\partial t^{2}}\right) \frac{\partial^{2} F}{\partial z^{2}} \\
-\frac{\sigma_{1} B_{0}^{2}}{\rho}\left(1+\lambda_{1} \frac{\partial}{\partial t}+\lambda_{2} \frac{\partial^{2}}{\partial t^{2}}\right) F \\
-\frac{v \phi}{k}\left(1+\lambda_{3} \frac{\partial}{\partial t}+\lambda_{4} \frac{\partial^{2}}{\partial t^{2}}\right) F
\end{array}\right],
$$

where $F=u+i v$ is the complex velocity of the fluid and $u$ and $v$ are its components in $x$ and $y$-directions, respectively, $\rho$ is the fluid density, $\mu$ is the dynamic viscosity, $v$ is the kinematic viscosity, $B_{0}$ is the magnitude of $\mathbf{B}_{0}, \sigma_{1}$ is the finite electrical conductivity of the fluid, $\phi(0<\phi<1)$ is the porosity and $k>0$ is the permeability of the porous medium, $\lambda_{1}$ and $\lambda_{3}$ are relaxation and retardation times, whereas $\lambda_{2}$ and $\lambda_{4}$ are material constants having the dimensions as the square of time. The corresponding boundary and initial conditions are

$$
\begin{aligned}
& F(0, t)=A t ; \quad F(\infty, t)=0 ; \quad t>0, \\
& F(z, 0)=\frac{\partial F(z, 0)}{\partial t}=\frac{\partial^{2} F(z, 0)}{\partial t^{2}}=0 ; \quad z>0,
\end{aligned}
$$

where $A$ with dimension $L / T^{2}$ is the constant acceleration of the plate in $x$-direction.

Introducing the following non-dimensional variables

$$
\xi=z\left(\frac{A}{v^{2}}\right)^{\frac{1}{3}} ; \quad \tau=t\left(\frac{A^{2}}{v}\right)^{\frac{1}{3}} ; \quad G=\frac{F}{(v A)^{\frac{1}{3}}},
$$


the dimensionless problem becomes

$$
\begin{aligned}
& \frac{\partial^{2} G(\xi, \tau)}{\partial \xi^{2}}+\gamma \frac{\partial^{3} G(\xi, \tau)}{\partial \tau \partial \xi^{2}}+\eta \frac{\partial^{4} G(\xi, \tau)}{\partial \tau^{2} \partial \xi^{2}}-\beta \frac{\partial^{3} G(\xi, \tau)}{\partial \tau^{3}} \\
& -a_{0} \frac{\partial^{2} G(\xi, \tau)}{\partial \tau^{2}}-b_{0} \frac{\partial G}{\partial \tau}-c_{0} G=0 ; \quad \xi, \tau>0, \\
& G(0, \tau)=\tau ; \quad G(\infty, \tau)=0 ; \quad \tau>0, \\
& G(\xi, 0)=\frac{\partial G(\xi, 0)}{\partial \tau}=\frac{\partial^{2} G(\xi, 0)}{\partial \tau^{2}}=0 ; \quad \xi>0,
\end{aligned}
$$

where

$$
\begin{aligned}
& \alpha=\lambda_{1}\left(\frac{A^{2}}{v}\right)^{\frac{1}{3}}, \quad \beta=\lambda_{2}\left(\frac{A^{2}}{v}\right)^{\frac{2}{3}}, \quad \gamma=\lambda_{3}\left(\frac{A^{2}}{v}\right)^{\frac{1}{3}}, \quad \eta=\lambda_{4}\left(\frac{A^{2}}{v}\right)^{\frac{2}{3}}, \\
& \omega=\Omega\left(\frac{v}{A^{2}}\right)^{\frac{1}{3}}, \quad M^{2}=\frac{\sigma_{1} B_{0}^{2} v^{\frac{1}{3}}}{\rho A^{\frac{2}{3}}}, \quad \frac{1}{K}=\frac{\phi \mu}{k \rho}\left(\frac{v}{A^{2}}\right)^{\frac{1}{3}}, \\
& c_{0}=2 i \omega+M^{2}+\frac{1}{K}, \quad a_{0}=\alpha+2 i \omega \beta+M^{2} \beta+\frac{\eta}{K} ; \quad b_{0}=1+2 i \omega \alpha+M^{2} \alpha+\frac{\gamma}{K} .
\end{aligned}
$$

Here $\alpha$ and $\gamma$ are the non-dimensional relaxation and retardation times, $\beta$ and $\eta$ are nondimensional material constants, $\omega$ is the non-dimensional angular velocity, $M^{2}$ is the nondimensional magnetic parameter called Hartmann number and $\frac{1}{K}$ is the non-dimensional porosity parameter, where the arbitrary constants $a_{0}, b_{0}$ and $c_{0}$ are introduced for the sake of mathematical convenience.

The solution of Eq. (5) in view of Eqs. (6) and (7) in the transformed $q$-plane is given by

$$
\bar{G}(\xi, q)=\frac{1}{q^{2}} \exp \left[-\sqrt{\frac{\beta q^{3}+a_{0} q^{2}+b_{0} q+c_{0}}{\eta q^{2}+\gamma q+1}} \xi\right],
$$

where

$$
\bar{G}(\xi, q)=\mathcal{L}\{G(\xi, \tau)\}=\int_{0}^{\infty} \exp (-q \tau) G(\xi, \tau) \mathrm{d} \tau .
$$

To find the Laplace inverse of Eq. (9), we use a similar procedure as in [22] and write $\bar{G}(\xi, q)$ in the product form as:

$$
\begin{aligned}
& \bar{G}(\xi, q)=\bar{G}_{1}(q) \bar{G}_{2}(\xi, q), \\
& \bar{G}_{1}(q)=\frac{1}{q^{2}}, \\
& \bar{G}_{2}(\xi, q)=\exp (-\xi \sqrt{w(q)}) ; \quad w(q)=\frac{\beta q^{3}+a_{0} q^{2}+b_{0} q+c_{0}}{\eta q^{2}+\gamma q+1} .
\end{aligned}
$$

Denoting $G_{1}(\tau)=\mathcal{L}^{-1}\left\{\bar{G}_{1}(q)\right\}$ and $G_{2}(\xi, \tau)=\mathcal{L}^{-1}\left\{\bar{G}_{2}(\xi, q)\right\}$, then by using the convolution theorem [23], we have

$$
G(\xi, \tau)=\left(G_{1} * G_{2}\right)(\tau)=\int_{0}^{\tau} G_{1}(\tau-s) G_{2}(\xi, s) \mathrm{d} s
$$


The Laplace inverse of Eq. (11) yields

$$
G_{1}(\tau)=\tau
$$

To find $G_{2}(\xi, \tau)=\mathcal{L}^{-1}\left\{\bar{G}_{2}(\xi, q)\right\}$, we use the inversion formula for compound functions [23]

$$
\mathcal{L}^{-1}\{F[w(q)]\}=\int_{0}^{\infty} f(u) g(u, \tau) \mathrm{d} u
$$

where $f(\tau)=\mathcal{L}^{-1}\{F(q)\}$ and $g(u, \tau)=\mathcal{L}^{-1}\{\exp (-u w(q))\}$.

Choosing $f(\xi, q)=\exp (-\xi \sqrt{q})$, we have

$$
\begin{aligned}
f(\xi, \tau)= & \mathcal{L}^{-1}\{\exp (-\xi \sqrt{q})\}=\frac{\xi}{2 \tau \sqrt{\pi \tau}} \exp \left(\frac{-\xi^{2}}{4 \tau}\right) ; \quad \xi>0, \\
G_{2}(\xi, \tau) & =\mathcal{L}^{-1}\left\{\bar{G}_{2}(\xi, q)\right\}=\int_{0}^{\infty} f(\xi, u) g(u, \tau) \mathrm{d} u \\
& =\frac{\xi}{2 \sqrt{\pi}} \int_{0}^{\infty} \frac{1}{u \sqrt{u}} \exp \left(\frac{-\xi^{2}}{4 u}\right) g(u, \tau) \mathrm{d} u .
\end{aligned}
$$

Now to find $g(u, \tau)=\mathcal{L}^{-1}\{\exp (-u w(q))\}$, we write $w(q)$ in the following form:

$$
w(q)=\frac{\beta q^{3}+a_{0} q^{2}+b_{0} q+c_{0}}{\eta q^{2}+\gamma q+1}=\eta_{0}+\frac{\beta}{\eta} q+\frac{\eta_{1}}{q-q_{1}}+\frac{\eta_{2}}{q-q_{2}},
$$

where

$$
\begin{aligned}
& \eta_{0}=\frac{a_{0}}{\eta}-\frac{\beta \gamma}{\eta^{2}} ; \quad \eta_{1}=\frac{\left(A_{1} q_{1}+A_{2}\right) \eta}{\sqrt{\gamma^{2}-4 \eta}} ; \quad \eta_{2}=-\frac{\left(A_{1} q_{2}+A_{2}\right) \eta}{\sqrt{\gamma^{2}-4 \eta}} \\
& A_{1}=b_{0}-\frac{\beta}{\eta}-\frac{a_{0} \gamma}{\eta}+\frac{\beta \gamma^{2}}{\eta^{2}} ; \quad A_{2}=c_{0}-\frac{a_{0}}{\eta}+\frac{\beta \gamma}{\eta^{2}}
\end{aligned}
$$

and $q_{1,2}=\frac{-\gamma \pm \sqrt{\gamma^{2}-4 \eta}}{2 \eta}$ are the roots of the equation $\eta q^{2}+\gamma q+1=0$.

Thus

$$
\begin{aligned}
g(u, \tau) & =\mathcal{L}^{-1}\left\{\exp \left(-u \eta_{0}\right) \exp \left(-\frac{u \beta}{\eta} q\right) \exp \left(-\frac{u \eta_{1}}{q-q_{1}}\right) \exp \left(-\frac{u \eta_{2}}{q-q_{2}}\right)\right\} \\
& =\exp \left(-u \eta_{0}\right) \mathcal{L}^{-1}\left\{\exp \left(-\frac{u \beta}{\eta} q\right)\left[1-H_{1}(q)-H_{2}(q)+H_{1}(q) H_{2}(q)\right]\right\},
\end{aligned}
$$

where $H_{1}(q)=1-\exp \left(-\frac{u \eta_{1}}{q-q_{1}}\right)$ and $H_{2}(q)=1-\exp \left(-\frac{u \eta_{2}}{q-q_{2}}\right)$.

If we denote

$$
\begin{aligned}
& h_{1}(\tau)=\mathcal{L}^{-1}\left\{H_{1}(q)\right\}=\sqrt{\frac{\eta_{1} u}{\tau}} \exp \left(q_{1} \tau\right) J_{1}\left(2 \sqrt{\eta_{1} u \tau}\right), \\
& h_{2}(\tau)=\mathcal{L}^{-1}\left\{H_{2}(q)\right\}=\sqrt{\frac{\eta_{2} u}{\tau}} \exp \left(q_{2} \tau\right) J_{1}\left(2 \sqrt{\eta_{2} u \tau}\right),
\end{aligned}
$$


where $J_{1}(\cdot)$ is the Bessel function of the first kind of order one, then finally one has

$$
\begin{aligned}
g(u, \tau)= & e^{-u \eta_{0}} \delta\left(\tau-\frac{\beta u}{\eta}\right)-\sqrt{\eta_{1} u} \int_{0}^{\tau} \frac{\delta\left(s-\frac{\beta u}{\eta}\right)}{\sqrt{\tau-s}} \exp \left(q_{1}(\tau-s)-u \eta_{0}\right) \\
& \times J_{1}\left(2 \sqrt{\eta_{1} u(\tau-s)}\right) \mathrm{d} s \\
& -\sqrt{\eta_{2} u} \int_{0}^{\tau} \frac{\delta\left(s-\frac{\beta u}{\eta}\right)}{\sqrt{\tau-s}} \exp \left(q_{2}(\tau-s)-u \eta_{0}\right) J_{1}\left(2 \sqrt{\eta_{2} u(\tau-s)}\right) \mathrm{d} s \\
& +u \sqrt{\eta_{1} \eta_{2}} \int_{0}^{\tau} \int_{0}^{\sigma} \frac{\delta\left(\tau-s-\frac{\beta u}{\eta}\right)}{\sqrt{\sigma(s-\sigma)}} \exp \left(q_{1} \sigma+q_{2}(s-\sigma)-u \eta_{0}\right) \\
& \times J_{1}\left(2 \sqrt{\eta_{1} u \sigma}\right) J_{1}\left(2 \sqrt{\eta_{2} u(s-\sigma)}\right) \mathrm{d} s \mathrm{~d} \sigma,
\end{aligned}
$$

where $\mathcal{L}^{-1}\{\exp (-\alpha q)\}=\delta(\tau-\alpha)$ and $\delta(\cdot)$ indicates the Dirac delta function.

Invoking Eq. (18) into Eq. (16), we get

$$
\begin{aligned}
G_{2}(\xi, \tau)= & \frac{\xi}{2 \sqrt{\pi}} \int_{0}^{\infty} \frac{\delta\left(\tau-\frac{\beta u}{\eta}\right)}{u \sqrt{u}} \exp \left(\frac{-\xi^{2}}{4 u}-\eta_{0} u\right) \mathrm{d} u-\frac{\xi \sqrt{\eta_{1}}}{2 \sqrt{\pi}} \int_{0}^{\tau} \int_{0}^{\infty} \frac{1}{u} \frac{\delta\left(s-\frac{\beta u}{\eta}\right)}{\sqrt{\tau-s}} \\
& \times \exp \left(\frac{-\xi^{2}}{4 u}+q_{1}(\tau-s)-\eta_{0} u\right) J_{1}\left(2 \sqrt{\eta_{1} u(\tau-s)}\right) \mathrm{d} u \mathrm{~d} s \\
& -\frac{\xi \sqrt{\eta_{2}}}{2 \sqrt{\pi}} \int_{0}^{\tau} \int_{0}^{\infty} \frac{\delta\left(s-\frac{\beta u}{\eta}\right)}{u \sqrt{(\tau-s)}} \exp \left(\frac{-\xi^{2}}{4 u}+q_{2}(\tau-s)-\eta_{0} u\right) \\
& \times J_{1}\left(2 \sqrt{\eta_{2} u(\tau-s)}\right) \mathrm{d} u \mathrm{~d} s \\
& +\frac{\xi \sqrt{\eta_{1} \eta_{2}}}{2 \sqrt{\pi}} \int_{0}^{\tau} \int_{0}^{\sigma} \int_{0}^{\infty} \frac{\delta\left(\tau-s-\frac{\beta u}{\eta}\right)}{\sqrt{u \sigma(s-\sigma)}} \exp \left(\frac{-\xi^{2}}{4 u}+q_{1} \sigma+q_{2}(s-\sigma)-\eta_{0} u\right) \\
& \times J_{1}\left(2 \sqrt{\eta_{1} u \sigma}\right) J_{1}\left(2 \sqrt{\eta_{2} u(s-\sigma)}\right) \mathrm{d} u \mathrm{~d} s \mathrm{~d} \sigma .
\end{aligned}
$$

Introducing Eqs. (19) and (14) into Eq. (13), we get

$$
\begin{aligned}
G(\xi, \tau)= & \frac{\xi}{2 \sqrt{\pi}} \int_{0}^{\tau} \int_{0}^{\infty} \frac{(\tau-s) \delta\left(s-\frac{\beta u}{\eta}\right)}{u \sqrt{u}} \exp \left(\frac{-\xi^{2}}{4 u}-\eta_{0} u\right) \mathrm{d} u \mathrm{~d} s \\
& -\frac{\xi \sqrt{\eta_{1}}}{2 \sqrt{\pi}} \int_{0}^{\tau} \int_{0}^{\sigma} \int_{0}^{\infty} \frac{(\tau-s) \delta\left(\sigma-\frac{\beta u}{\eta}\right)}{u \sqrt{s-\sigma}} \exp \left(\frac{-\xi^{2}}{4 u}+q_{1}(s-\sigma)-\eta_{0} u\right) \\
& \times J_{1}\left(2 \sqrt{\eta_{1} u(s-\sigma)}\right) \mathrm{d} u \mathrm{~d} s \mathrm{~d} \sigma \\
& -\frac{\xi \sqrt{\eta_{2}}}{2 \sqrt{\pi}} \int_{0}^{\tau} \int_{0}^{\sigma} \int_{0}^{\infty} \frac{(\tau-s) \delta\left(\sigma-\frac{\beta u}{\eta}\right)}{u \sqrt{s-\sigma}} J_{1}\left(2 \sqrt{\eta_{2} u(s-\sigma)}\right) \\
& \times \exp \left(\frac{-\xi^{2}}{4 u}+q_{2}(s-\sigma)-\eta_{0} u\right) \mathrm{d} u \mathrm{~d} s \mathrm{~d} \sigma \\
& +\frac{\xi \sqrt{\eta_{1} \eta_{2}}}{2 \sqrt{\pi}} \int_{0}^{\tau} \int_{0}^{\sigma} \int_{0}^{w} \int_{0}^{\infty} \frac{(\tau-s) \delta\left(s-\sigma-\frac{\beta u}{\eta}\right)}{\sqrt{u w(\sigma-w)}} \\
& \times \exp \left(\frac{-\xi^{2}}{4 u}+q_{1} w+q_{2}(s-\sigma)-\eta_{0} u\right) J_{1}\left(2 \sqrt{\eta_{1} u w}\right) \\
& \times J_{1}\left(2 \sqrt{\eta_{2} u(\sigma-w)}\right) \mathrm{d} u \mathrm{~d} s \mathrm{~d} \sigma \mathrm{d} w .
\end{aligned}
$$


Taking $u=\frac{v \eta}{\beta}$ into Eq. (20) and keeping in mind

$$
\int_{a}^{b} f(x) \delta\left(x-x_{0}\right) \mathrm{d} x= \begin{cases}f\left(x_{0}\right) & \text { for } x \in[a, b), \\ 0 & \text { for } x \notin[a, b),\end{cases}
$$

one finally obtains

$$
\begin{aligned}
G(\xi, \tau)= & \frac{\xi \sqrt{\beta}}{2 \sqrt{\pi \eta}} \int_{0}^{\tau} \frac{(\tau-s)}{s \sqrt{s}} \exp \left(\frac{-\beta \xi^{2}}{4 \eta s}-\frac{\eta \eta_{0}}{\beta} s\right) \mathrm{d} s \\
& -\frac{\xi \sqrt{\eta_{1}}}{2 \sqrt{\pi}} \int_{0}^{\tau} \int_{0}^{\sigma} \frac{(\tau-s)}{\sigma \sqrt{s-\sigma}} \exp \left(\frac{-\beta \xi^{2}}{4 \eta \sigma}+q_{1}(s-\sigma)-\frac{\eta_{0} \eta}{\beta} \sigma\right) \\
& \times J_{1}\left(2 \sqrt{\frac{\eta_{1} \eta}{\beta} \sigma(s-\sigma)}\right) \mathrm{d} s \mathrm{~d} \sigma \\
& -\frac{\xi \sqrt{\eta_{2}}}{2 \sqrt{\pi}} \int_{0}^{\tau} \int_{0}^{\sigma} \frac{(\tau-s)}{\sigma \sqrt{s-\sigma}} \exp \left(\frac{-\beta \xi^{2}}{4 \eta \sigma}+q_{2}(s-\sigma)-\frac{\eta_{0} \eta}{\beta} \sigma\right) \\
& \times J_{1}\left(2 \sqrt{\frac{\eta_{2} \eta}{\beta} \sigma(s-\sigma)}\right) \mathrm{d} s \mathrm{~d} \sigma \\
& +\frac{\xi \sqrt{\eta_{1} \eta_{2} \eta}}{2 \sqrt{\beta \pi}} \int_{0}^{\tau} \int_{0}^{\sigma} \int_{0}^{w} \frac{(\tau-s)}{\sqrt{w(s-\sigma)(\sigma-w)}} \\
& \times \exp \left(\frac{-\beta \xi^{2}}{4 \eta(s-\sigma)}+q_{1} w+q_{2}(s-\sigma)-\frac{\eta_{0} \eta}{\beta}(s-\sigma)\right) \\
& \times J_{1}\left(2 \sqrt{\frac{\eta_{1} \eta}{\beta} w(s-\sigma)}\right) J_{1}\left(2 \sqrt{\frac{\eta_{2} \eta}{\beta}(s-\sigma)(\sigma-w)}\right) \mathrm{d} s \mathrm{~d} \sigma \mathrm{d} w .
\end{aligned}
$$

The starting solution (22) holds for both small and large times. In order to write as a sum of the steady-state and transient solutions, we use the relation

$$
\int_{0}^{\tau} f(\xi, \tau, s) \mathrm{d} s=\int_{0}^{\infty} f(\xi, \tau, s) \mathrm{d} s-\int_{\tau}^{\infty} f(\xi, \tau, s) \mathrm{d} s .
$$

Hence Eq. (22) reduces to the following form:

$$
\begin{aligned}
G(\xi, \tau)= & \tau \exp \left(-\xi \sqrt{\eta_{0}}\right)-\frac{\xi \beta}{2 \eta \sqrt{\eta_{0}}} \exp \left(-\xi \sqrt{\eta_{0}}\right) \\
& -\frac{\xi \sqrt{\beta}}{2 \sqrt{\pi \eta}} \int_{\tau}^{\infty} \frac{(\tau-s)}{s \sqrt{s}} \exp \left(\frac{-\beta \xi^{2}}{4 \eta s}-\frac{\eta \eta_{0}}{\beta} s\right) \mathrm{d} s \\
& -\frac{\xi \sqrt{\eta_{1}}}{2 \sqrt{\pi}} \int_{0}^{\tau} \int_{0}^{\sigma} \frac{(\tau-s)}{\sigma \sqrt{s-\sigma}} \exp \left(\frac{-\beta \xi^{2}}{4 \eta \sigma}+q_{1}(s-\sigma)-\frac{\eta_{0} \eta}{\beta} \sigma\right) \\
& \times J_{1}\left(2 \sqrt{\frac{\eta_{1} \eta}{\beta} \sigma(s-\sigma)}\right) \mathrm{d} s \mathrm{~d} \sigma \\
& -\frac{\xi \sqrt{\eta_{2}}}{2 \sqrt{\pi}} \int_{0}^{\tau} \int_{0}^{\sigma} \frac{(\tau-s)}{\sigma \sqrt{s-\sigma}} \exp \left(\frac{-\beta \xi^{2}}{4 \eta \sigma}+q_{2}(s-\sigma)-\frac{\eta_{0} \eta}{\beta} \sigma\right) \\
& \times J_{1}\left(2 \sqrt{\frac{\eta_{2} \eta}{\beta}} \sigma(s-\sigma)\right) \mathrm{d} s \mathrm{~d} \sigma \\
& +\frac{\xi \sqrt{\eta_{1} \eta_{2} \eta}}{2 \sqrt{\beta \pi}} \int_{0}^{\tau} \int_{0}^{\sigma} \int_{0}^{w} \frac{(\tau-s)}{\sqrt{w(s-\sigma)(\sigma-w)}}
\end{aligned}
$$




$$
\begin{aligned}
& \times \exp \left(\frac{-\beta \xi^{2}}{4 \eta(s-\sigma)}+q_{1} w+q_{2}(s-\sigma)-\frac{\eta_{0} \eta}{\beta}(s-\sigma)\right) \\
& \times J_{1}\left(2 \sqrt{\frac{\eta_{1} \eta}{\beta} w(s-\sigma)}\right) J_{1}\left(2 \sqrt{\frac{\eta_{2} \eta}{\beta}(s-\sigma)(\sigma-w)}\right) \mathrm{d} s \mathrm{~d} \sigma \mathrm{d} w
\end{aligned}
$$

\section{Flow due to a variably accelerated plate}

Now let us consider the flow situation in which the motion in the fluid is induced due to a variably accelerated plate. The rest of the problem is the same except for the first boundary condition $(2)_{1}$ which is replaced by

$$
F(0, t)=B t^{2} ; \quad t>0
$$

in which $B$ with dimension $L / T^{3}$ denotes the variable acceleration of the plate in $x$-direction. In this case we introduce the following dimensionless variables

$$
\xi=z\left(\frac{B}{v^{3}}\right)^{\frac{1}{5}} ; \quad \tau=t\left(\frac{B^{2}}{v}\right)^{\frac{1}{5}} ; \quad G=\frac{F}{\left(v^{2} B\right)^{\frac{1}{5}}}
$$

into Eq. (1) along with boundary and initial conditions $(2)_{2},(25)$ and (3). Hence, finally, we arrive at the following dimensionless system of equations:

$$
\begin{aligned}
& \frac{\partial^{2} G(\xi, \tau)}{\partial \xi^{2}}+\gamma^{*} \frac{\partial^{3} G(\xi, \tau)}{\partial \tau \xi^{2}}+\eta^{*} \frac{\partial^{4} G(\xi, \tau)}{\partial \tau^{2} \partial \xi^{2}}-\beta^{*} \frac{\partial^{3} G(\xi, \tau)}{\partial \tau^{3}} \\
& -a_{0}^{*} \frac{\partial^{2} G(\xi, \tau)}{\partial \tau^{2}}-b_{0}^{*} \frac{\partial G}{\partial \tau}-c_{0}^{*} G=0 ; \quad \xi, \tau>0, \\
& G(0, \tau)=\tau^{2} ; \quad G(\infty, \tau)=0 ; \quad \tau>0, \\
& G(\xi, 0)=\frac{\partial G(\xi, 0)}{\partial \tau}=\frac{\partial^{2} G(\xi, 0)}{\partial \tau^{2}}=0 ; \quad \xi>0,
\end{aligned}
$$

where

$$
\begin{aligned}
& \alpha^{*}=\lambda_{1}\left(\frac{B^{2}}{v}\right)^{\frac{1}{5}}, \quad \beta^{*}=\lambda_{2}\left(\frac{B^{4}}{v^{2}}\right)^{\frac{1}{5}}, \quad \gamma^{*}=\lambda_{3}\left(\frac{B^{2}}{v}\right)^{\frac{1}{5}}, \\
& \eta^{*}=\lambda_{4}\left(\frac{B^{4}}{v^{2}}\right)^{\frac{1}{5}}, \quad \omega^{*}=\Omega\left(\frac{v}{B^{2}}\right)^{\frac{1}{5}}, \quad M^{*}=\frac{\sigma_{1} B_{0}^{2} v^{\frac{1}{5}}}{\rho A^{\frac{2}{5}}}, \\
& \frac{1}{K^{*}}=\frac{\phi v^{\frac{6}{5}}}{k B^{\frac{2}{5}}}, \quad c_{0}^{*}=2 i \omega^{*}+M^{* 2}+\frac{1}{K^{*}}, \\
& a_{0}^{*}=\alpha^{*}+2 i \omega \beta^{*}+M^{* 2} \beta^{*}+\frac{\eta^{*}}{K^{*}} ; \quad b_{0}^{*}=1+2 i \omega^{*} \alpha^{*}+M^{* 2} \alpha^{*}+\frac{\gamma^{*}}{K^{*}} .
\end{aligned}
$$

Following a similar methodology of solution as for a constantly accelerated plate, finally we arrive at the following solution (asterisk signs are dropped for mathematical simplicity):

$$
\begin{aligned}
G(\xi, \tau)= & \frac{\xi \sqrt{\beta}}{2 \sqrt{\pi \eta}} \int_{0}^{\tau} \frac{(\tau-s)^{2}}{s \sqrt{s}} \exp \left(\frac{-\beta \xi^{2}}{4 \eta s}-\frac{\eta \eta_{0}}{\beta} s\right) \mathrm{d} s \\
& -\frac{\xi \sqrt{\eta_{1}}}{2 \sqrt{\pi}} \int_{0}^{\tau} \int_{0}^{\sigma} \frac{(\tau-s)^{2}}{\sigma \sqrt{s-\sigma}} \exp \left(\frac{-\beta \xi^{2}}{4 \eta \sigma}+q_{1}(s-\sigma)-\frac{\eta_{0} \eta}{\beta} \sigma\right)
\end{aligned}
$$




$$
\begin{aligned}
& \times J_{1}\left(2 \sqrt{\frac{\eta_{1} \eta}{\beta} \sigma(s-\sigma)}\right) \mathrm{d} s \mathrm{~d} \sigma \\
& -\frac{\xi \sqrt{\eta_{2}}}{2 \sqrt{\pi}} \int_{0}^{\tau} \int_{0}^{\sigma} \frac{(\tau-s)^{2}}{\sigma \sqrt{s-\sigma}} \exp \left(\frac{-\beta \xi^{2}}{4 \eta \sigma}+q_{2}(s-\sigma)-\frac{\eta_{0} \eta}{\beta} \sigma\right) \\
& \times J_{1}\left(2 \sqrt{\frac{\eta_{2} \eta}{\beta} \sigma(s-\sigma)}\right) \mathrm{d} s \mathrm{~d} \sigma \\
& +\frac{\xi \sqrt{\eta_{1} \eta_{2} \eta}}{2 \sqrt{\beta \pi}} \int_{0}^{\tau} \int_{0}^{\sigma} \int_{0}^{w} \frac{(\tau-s)^{2}}{\sqrt{w(s-\sigma)(\sigma-w)}} \\
& \times \exp \left(\frac{-\beta \xi^{2}}{4 \eta(s-\sigma)}+q_{1} w+q_{2}(s-\sigma)-\frac{\eta_{0} \eta}{\beta}(s-\sigma)\right) \\
& \times J_{1}\left(2 \sqrt{\frac{\eta_{1} \eta}{\beta} w(s-\sigma)}\right) J_{1}\left(2 \sqrt{\frac{\eta_{2} \eta}{\beta}(s-\sigma)(\sigma-w)}\right) \mathrm{d} s \mathrm{~d} \sigma \mathrm{d} w .
\end{aligned}
$$

The corresponding solutions for small and large times are given by

$$
\begin{aligned}
& G(\xi, \tau)=\tau^{2} \exp \left(-\xi \sqrt{\eta_{0}}\right)-\frac{\xi \beta^{2}}{4 \eta\left(\eta_{0} \eta\right)^{\frac{3}{2}}} \exp \left(-\xi \sqrt{\eta_{0}}\right)\left(1+\xi \sqrt{\eta_{0}}\right)-\frac{\tau \xi \beta}{\eta \sqrt{\eta_{0}}} \exp \left(-\xi \sqrt{\eta_{0}}\right) \\
& -\frac{\xi \sqrt{\beta}}{2 \sqrt{\pi \eta}} \int_{\tau}^{\infty} \frac{(\tau-s)^{2}}{s \sqrt{s}} \exp \left(\frac{-\beta \xi^{2}}{4 \eta s}-\frac{\eta \eta_{0}}{\beta} s\right) \mathrm{d} s \\
& -\frac{\xi \sqrt{\eta_{1}}}{2 \sqrt{\pi}} \int_{0}^{\tau} \int_{0}^{\sigma} \frac{(\tau-s)^{2}}{\sigma \sqrt{s-\sigma}} \exp \left(\frac{-\beta \xi^{2}}{4 \eta \sigma}+q_{1}(s-\sigma)-\frac{\eta_{0} \eta}{\beta} \sigma\right) \\
& \times J_{1}\left(2 \sqrt{\frac{\eta_{1} \eta}{\beta} \sigma(s-\sigma)}\right) \mathrm{d} s \mathrm{~d} \sigma \\
& -\frac{\xi \sqrt{\eta_{2}}}{2 \sqrt{\pi}} \int_{0}^{\tau} \int_{0}^{\sigma} \frac{(\tau-s)^{2}}{\sigma \sqrt{s-\sigma}} \exp \left(\frac{-\beta \xi^{2}}{4 \eta \sigma}+q_{2}(s-\sigma)-\frac{\eta_{0} \eta}{\beta} \sigma\right) \\
& \times J_{1}\left(2 \sqrt{\frac{\eta_{2} \eta}{\beta} \sigma(s-\sigma)}\right) \mathrm{d} s \mathrm{~d} \sigma \\
& +\frac{\xi \sqrt{\eta_{1} \eta_{2} \eta}}{2 \sqrt{\beta \pi}} \int_{0}^{\tau} \int_{0}^{\sigma} \int_{0}^{w} \frac{(\tau-s)^{2}}{\sqrt{w(s-\sigma)(\sigma-w)}} \\
& \times \exp \left(\frac{-\beta \xi^{2}}{4 \eta(s-\sigma)}+q_{1} w+q_{2}(s-\sigma)-\frac{\eta_{0} \eta}{\beta}(s-\sigma)\right) \\
& \times J_{1}\left(2 \sqrt{\frac{\eta_{1} \eta}{\beta} w(s-\sigma)}\right) J_{1}\left(2 \sqrt{\frac{\eta_{2} \eta}{\beta}(s-\sigma)(\sigma-w)}\right) \mathrm{d} s \mathrm{~d} \sigma \mathrm{d} w
\end{aligned}
$$

It is important to note that the obtained solutions (24) and (32) clearly satisfy the imposed boundary and initial conditions, which serves as a validation of the presented results.

\section{Limiting cases}

In this section we reduce our solutions to their limiting cases.

(i) The above solutions for a hydrodynamic fluid $(M=0)$ in a non-porous medium $(1 / K=$ 0 ) result in

$$
\begin{aligned}
G(\xi, \tau)= & \frac{\xi \sqrt{\beta}}{2 \sqrt{\pi \eta}} \int_{0}^{\tau} \frac{(\tau-s)}{s \sqrt{s}} \exp \left(\frac{-\beta \xi^{2}}{4 \eta s}-\frac{\eta \eta_{3}}{\beta} s\right) \mathrm{d} s \\
& -\frac{\xi \sqrt{\eta_{4}}}{2 \sqrt{\pi}} \int_{0}^{\tau} \int_{0}^{\sigma} \frac{(\tau-s)}{\sigma \sqrt{s-\sigma}} \exp \left(\frac{-\beta \xi^{2}}{4 \eta \sigma}+q_{1}(s-\sigma)-\frac{\eta_{3} \eta}{\beta} \sigma\right)
\end{aligned}
$$




$$
\begin{aligned}
& \times J_{1}\left(2 \sqrt{\frac{\eta_{4} \eta}{\beta} \sigma(s-\sigma)}\right) \mathrm{d} s \mathrm{~d} \sigma \\
& -\frac{\xi \sqrt{\eta_{5}}}{2 \sqrt{\pi}} \int_{0}^{\tau} \int_{0}^{\sigma} \frac{(\tau-s)}{\sigma \sqrt{s-\sigma}} \exp \left(\frac{-\beta \xi^{2}}{4 \eta \sigma}+q_{2}(s-\sigma)-\frac{\eta_{3} \eta}{\beta} \sigma\right) \\
& \times J_{1}\left(2 \sqrt{\frac{\eta_{5} \eta}{\beta} \sigma(s-\sigma)}\right) \mathrm{d} s \mathrm{~d} \sigma \\
& +\frac{\xi \sqrt{\eta_{4} \eta_{5} \eta}}{2 \sqrt{\beta \pi}} \int_{0}^{\tau} \int_{0}^{\sigma} \int_{0}^{w} \frac{(\tau-s)}{\sqrt{w(s-\sigma)(\sigma-w)}} \\
& \times \exp \left(\frac{-\beta \xi^{2}}{4 \eta(s-\sigma)}+q_{1} w+q_{2}(s-\sigma)-\frac{\eta_{3} \eta}{\beta}(s-\sigma)\right) \\
& \times J_{1}\left(2 \sqrt{\frac{\eta_{4} \eta}{\beta} w(s-\sigma)}\right) J_{1}\left(2 \sqrt{\frac{\eta_{5} \eta}{\beta}(s-\sigma)(\sigma-w)}\right) \mathrm{d} s \mathrm{~d} \sigma \mathrm{d} w
\end{aligned}
$$

respectively

$$
\begin{aligned}
G(\xi, \tau)= & \frac{\xi \sqrt{\beta}}{2 \sqrt{\pi \eta}} \int_{0}^{\tau} \frac{(\tau-s)^{2}}{s \sqrt{s}} \exp \left(\frac{-\beta \xi^{2}}{4 \eta s}-\frac{\eta \eta_{3}}{\beta} s\right) \mathrm{d} s \\
& -\frac{\xi \sqrt{\eta_{4}}}{2 \sqrt{\pi}} \int_{0}^{\tau} \int_{0}^{\sigma} \frac{(\tau-s)^{2}}{\sigma \sqrt{s-\sigma}} \exp \left(\frac{-\beta \xi^{2}}{4 \eta \sigma}+q_{1}(s-\sigma)-\frac{\eta_{3} \eta}{\beta} \sigma\right) \\
& \times J_{1}\left(2 \sqrt{\left.\frac{\eta_{4} \eta}{\beta} \sigma(s-\sigma)\right)} \mathrm{d} s \mathrm{~d} \sigma\right. \\
& -\frac{\xi \sqrt{\eta_{5}}}{2 \sqrt{\pi}} \int_{0}^{\tau} \int_{0}^{\sigma} \frac{(\tau-s)^{2}}{\sigma \sqrt{s-\sigma}} \exp \left(\frac{-\beta \xi^{2}}{4 \eta \sigma}+q_{2}(s-\sigma)-\frac{\eta_{3} \eta}{\beta} \sigma\right) \\
& \times J_{1}\left(2 \sqrt{\left.\frac{\eta_{5} \eta}{\beta} \sigma(s-\sigma)\right)} \mathrm{d} s \mathrm{~d} \sigma\right. \\
& +\frac{\xi \sqrt{\eta_{4} \eta_{5} \eta}}{2 \sqrt{\beta \pi}} \int_{0}^{\tau} \int_{0}^{\sigma} \int_{0}^{w} \frac{(\tau-s)^{2}}{\sqrt{w(s-\sigma)(\sigma-w)}} \\
& \times \exp \left(\frac{-\beta \xi^{2}}{4 \eta(s-\sigma)}+q_{1} w+q_{2}(s-\sigma)-\frac{\eta_{3} \eta}{\beta}(s-\sigma)\right) \\
& \times J_{1}\left(2 \sqrt{\frac{\eta_{4} \eta}{\beta} w(s-\sigma)}\right) J_{1}\left(2 \sqrt{\frac{\eta_{5} \eta}{\beta}(s-\sigma)(\sigma-w)}\right) \mathrm{d} s \mathrm{~d} \sigma \mathrm{d} w
\end{aligned}
$$

where

$$
\begin{aligned}
& \eta_{3}=\frac{\alpha+2 i \omega \beta}{\eta}-\frac{\beta \gamma}{\eta^{2}} ; \quad \eta_{4}=\frac{\left(A_{3} q_{1}+A_{4}\right) \eta}{\sqrt{\gamma^{2}-4 \eta}} ; \quad \eta_{5}=-\frac{\left(A_{3} q_{2}+A_{4}\right) \eta}{\sqrt{\gamma^{2}-4 \eta}} \\
& A_{3}=1+2 i \omega \alpha-\frac{\beta}{\eta}-\frac{(\alpha+2 i \omega \beta) \gamma}{\eta}+\frac{\beta \gamma^{2}}{\eta^{2}} \\
& A_{4}=2 i \omega-\frac{(\alpha+2 i \omega \beta)}{\eta}+\frac{\beta \gamma}{\eta^{2}} .
\end{aligned}
$$

It is important to note that although solutions (33) and (34) are obtained as a limiting case of solutions (22) and (31), these solutions are still new and are not reported in the existing literature. 
(ii) Making $\omega=0$ into Eqs. (22) and (31), similar solutions for a generalized Burgers? fluid in a non-rotating frame are obtained as follows:

$$
\begin{aligned}
& G(\xi, \tau)=\frac{\xi \sqrt{\beta}}{2 \sqrt{\pi \eta}} \int_{0}^{\tau} \frac{(\tau-s)}{s \sqrt{s}} \exp \left(\frac{-\beta \xi^{2}}{4 \eta s}-\frac{\eta \eta_{6}}{\beta} s\right) \mathrm{d} s \\
& -\frac{\xi \sqrt{\eta_{7}}}{2 \sqrt{\pi}} \int_{0}^{\tau} \int_{0}^{\sigma} \frac{(\tau-s)}{\sigma \sqrt{s-\sigma}} \exp \left(\frac{-\beta \xi^{2}}{4 \eta \sigma}+q_{1}(s-\sigma)-\frac{\eta_{6} \eta}{\beta} \sigma\right) \\
& \times J_{1}\left(2 \sqrt{\frac{\eta_{7} \eta}{\beta} \sigma(s-\sigma)}\right) \mathrm{d} s \mathrm{~d} \sigma \\
& -\frac{\xi \sqrt{\eta_{8}}}{2 \sqrt{\pi}} \int_{0}^{\tau} \int_{0}^{\sigma} \frac{(\tau-s)}{\sigma \sqrt{s-\sigma}} \exp \left(\frac{-\beta \xi^{2}}{4 \eta \sigma}+q_{2}(s-\sigma)-\frac{\eta_{6} \eta}{\beta} \sigma\right) \\
& \times J_{1}\left(2 \sqrt{\frac{\eta_{8} \eta}{\beta} \sigma(s-\sigma)}\right) \mathrm{d} s \mathrm{~d} \sigma \\
& +\frac{\xi \sqrt{\eta_{7} \eta_{8} \eta}}{2 \sqrt{\beta \pi}} \int_{0}^{\tau} \int_{0}^{\sigma} \int_{0}^{w} \frac{(\tau-s)}{\sqrt{w(s-\sigma)(\sigma-w)}} \\
& \times \exp \left(\frac{-\beta \xi^{2}}{4 \eta(s-\sigma)}+q_{1} w+q_{2}(s-\sigma)-\frac{\eta_{6} \eta}{\beta}(s-\sigma)\right) \\
& \times J_{1}\left(2 \sqrt{\frac{\eta_{7} \eta}{\beta} w(s-\sigma)}\right) J_{1}\left(2 \sqrt{\frac{\eta_{8} \eta}{\beta}(s-\sigma)(\sigma-w)}\right) \mathrm{d} s \mathrm{~d} \sigma \mathrm{d} w ; \\
& G(\xi, \tau)=\frac{\xi \sqrt{\beta}}{2 \sqrt{\pi \eta}} \int_{0}^{\tau} \frac{(\tau-s)^{2}}{s \sqrt{s}} \exp \left(\frac{-\beta \xi^{2}}{4 \eta s}-\frac{\eta \eta_{6}}{\beta} s\right) \mathrm{d} s \\
& -\frac{\xi \sqrt{\eta_{7}}}{2 \sqrt{\pi}} \int_{0}^{\tau} \int_{0}^{\sigma} \frac{(\tau-s)^{2}}{\sigma \sqrt{s-\sigma}} \exp \left(\frac{-\beta \xi^{2}}{4 \eta \sigma}+q_{1}(s-\sigma)-\frac{\eta_{6} \eta}{\beta} \sigma\right) \\
& \times J_{1}\left(2 \sqrt{\frac{\eta_{7} \eta}{\beta} \sigma(s-\sigma)}\right) \mathrm{d} s \mathrm{~d} \sigma \\
& -\frac{\xi \sqrt{\eta_{8}}}{2 \sqrt{\pi}} \int_{0}^{\tau} \int_{0}^{\sigma} \frac{(\tau-s)^{2}}{\sigma \sqrt{s-\sigma}} \exp \left(\frac{-\beta \xi^{2}}{4 \eta \sigma}+q_{2}(s-\sigma)-\frac{\eta_{6} \eta}{\beta} \sigma\right) \\
& \times J_{1}\left(2 \sqrt{\frac{\eta_{8} \eta}{\beta} \sigma(s-\sigma)}\right) \mathrm{d} s \mathrm{~d} \sigma \\
& +\frac{\xi \sqrt{\eta_{7} \eta_{8} \eta}}{2 \sqrt{\beta \pi}} \int_{0}^{\tau} \int_{0}^{\sigma} \int_{0}^{w} \frac{(\tau-s)^{2}}{\sqrt{w(s-\sigma)(\sigma-w)}} \\
& \times \exp \left(\frac{-\beta \xi^{2}}{4 \eta(s-\sigma)}+q_{1} w+q_{2}(s-\sigma)-\frac{\eta_{6} \eta}{\beta}(s-\sigma)\right) \\
& \times J_{1}\left(2 \sqrt{\frac{\eta_{7} \eta}{\beta} w(s-\sigma)}\right) J_{1}\left(2 \sqrt{\frac{\eta_{8} \eta}{\beta}(s-\sigma)(\sigma-w)}\right) \mathrm{d} s \mathrm{~d} \sigma \mathrm{d} w
\end{aligned}
$$

in which

$$
\begin{aligned}
& \eta_{6}=\frac{\alpha+M^{2} \beta+\frac{\eta}{K}}{\eta}-\frac{\beta \gamma}{\eta^{2}} ; \quad \eta_{7}=\frac{\left(A_{5} q_{1}+A_{6}\right) \eta}{\sqrt{\gamma^{2}-4 \eta}} ; \quad \eta_{8}=-\frac{\left(A_{5} q_{2}+A_{6}\right) \eta}{\sqrt{\gamma^{2}-4 \eta}}, \\
& A_{5}=1+M^{2} \alpha+\frac{\gamma}{K}-\frac{\beta}{\eta}-\frac{\left(\alpha+M^{2} \beta+\frac{\eta}{K}\right) \gamma}{\eta}+\frac{\beta \gamma^{2}}{\eta^{2}} ; \\
& A_{6}=M^{2}+\frac{1}{K}-\frac{\alpha+M^{2} \beta+\frac{\eta}{K}}{\eta}+\frac{\beta \gamma}{\eta^{2}} .
\end{aligned}
$$


(iii) Making $\lambda_{4}$ and $\lambda_{2}=0$ into Eq. (12) and following the same way as before, we get the velocity field for an Oldroyd-B fluid performing the same motion

$$
\begin{aligned}
G(\xi, \tau)= & \frac{\xi \sqrt{\alpha}}{2 \sqrt{\pi \gamma}} \int_{0}^{\tau} \frac{(\tau-s)}{s \sqrt{s}} \exp \left(\frac{-\alpha \xi^{2}}{4 \gamma s}-\frac{\eta_{9} \gamma}{\alpha} s\right) \mathrm{d} s-\frac{\xi \sqrt{\eta_{11}}}{2 \sqrt{\pi}} \int_{0}^{\tau} \int_{0}^{\sigma} \frac{(\tau-s)}{\sigma \sqrt{s-\sigma}} \\
& \times \exp \left(\frac{-\alpha \xi^{2}}{4 \gamma \sigma}-\frac{\eta_{9} \gamma \sigma}{\alpha}-\gamma_{0}(s-\sigma)\right) J_{1}\left(2 \sqrt{\frac{\eta_{11} \gamma}{\alpha} \sigma(s-\sigma)}\right) \mathrm{d} s \mathrm{~d} \sigma,
\end{aligned}
$$

respectively

$$
\begin{aligned}
G(\xi, \tau)= & \frac{\xi \sqrt{\alpha}}{2 \sqrt{\pi \gamma}} \int_{0}^{\tau} \frac{(\tau-s)^{2}}{s \sqrt{s}} \exp \left(\frac{-\alpha \xi^{2}}{4 \gamma s}-\frac{\eta_{9} \gamma}{\alpha} s\right) \mathrm{d} s-\frac{\xi \sqrt{\eta_{11}}}{2 \sqrt{\pi}} \int_{0}^{\tau} \int_{0}^{\sigma} \frac{(\tau-s)^{2}}{\sigma \sqrt{s-\sigma}} \\
& \times \exp \left(\frac{-\alpha \xi^{2}}{4 \gamma \sigma}-\frac{\eta_{9} \gamma \sigma}{\alpha}-\gamma_{0}(s-\sigma)\right) J_{1}\left(2 \sqrt{\frac{\eta_{11} \gamma}{\alpha} \sigma(s-\sigma)}\right) \mathrm{d} s \mathrm{~d} \sigma,
\end{aligned}
$$

where

$$
\begin{aligned}
& \eta_{9}=\frac{1+2 i \omega \alpha+M^{2} \alpha+\frac{\gamma}{K}}{\gamma}-\frac{\alpha}{\gamma^{2}}, \quad \gamma_{0}=-\frac{1}{\gamma}, \quad \eta_{11}=\frac{\eta_{10}}{\gamma} \\
& \eta_{10}=2 i \omega+M^{2}+\frac{1}{K}-\left(1+2 i \omega \alpha+M^{2} \alpha+\frac{\gamma}{K}-\frac{\alpha}{\gamma}\right) \frac{1}{\gamma} .
\end{aligned}
$$

(iv) Now by letting $\lambda_{1}, \lambda_{2}, \lambda_{4}=0$ into Eq. (12), we get the velocity field for a second grade fluid performing the same motion

$$
\begin{aligned}
G_{c}(\xi, \tau)= & \tau \exp \left(-\xi \sqrt{\frac{b_{0}}{\gamma}}\right)+\frac{\sqrt{a_{1} \xi}}{2 \sqrt{\gamma \pi}} \int_{0}^{\infty} \int_{0}^{\infty} \frac{(\tau-s)}{u \sqrt{s}} \\
& \times \exp \left(-\frac{\xi^{2}}{4 \gamma u}-b_{0} u-\gamma_{0} s\right) I_{1}\left(2 \sqrt{a_{1} u s}\right) \mathrm{d} u \mathrm{~d} s \\
& +\frac{\sqrt{a_{1} \xi}}{2 \sqrt{\gamma \pi}} \int_{\tau}^{\infty} \int_{0}^{\infty} \frac{(\tau-s)}{u \sqrt{s}} \exp \left(-\frac{\xi^{2}}{4 \gamma u}-b_{0} u-\gamma_{0} s\right) I_{1}\left(2 \sqrt{a_{1} u s}\right) \mathrm{d} u \mathrm{~d} s \\
G_{v}(\xi, \tau)= & \tau^{2} \exp \left(-\xi \sqrt{\frac{b_{0}}{\gamma}}\right)+\frac{\sqrt{a_{1} \xi}}{2 \sqrt{\gamma \pi}} \int_{0}^{\infty} \int_{0}^{\infty} \frac{(\tau-s)^{2}}{u \sqrt{s}} \\
& \times \exp \left(-\frac{\xi^{2}}{4 \gamma u}-b_{0} u-\gamma_{0} s\right) I_{1}\left(2 \sqrt{a_{1} u s}\right) \mathrm{d} u \mathrm{~d} s \\
& +\frac{\sqrt{a_{1} \xi}}{2 \sqrt{\gamma \pi}} \int_{\tau}^{\infty} \int_{0}^{\infty} \frac{(\tau-s)}{u \sqrt{s}} \exp \left(-\frac{\xi^{2}}{4 \gamma u}-b_{0} u-\gamma_{0} s\right) I_{1}\left(2 \sqrt{a_{1} u s}\right) \mathrm{d} u \mathrm{~d} s
\end{aligned}
$$

where $I_{1}(\cdot)$ is the modified Bessel function of the first kind of order one and

$$
b_{0}=1+\frac{\gamma}{K}, \quad c_{0}=2 i \omega+M^{2}+\frac{1}{K}, \quad a_{1}=\gamma_{0} b_{0}-c_{0}, \quad \gamma_{0}=\frac{1}{\gamma} .
$$

The above solutions (39) and (40) are in accordance to those obtained in [22] (see Eqs. (23) and (28)). 
(v) Finally, making $\lambda_{1}, \lambda_{2}, \lambda_{3}$ and $\lambda_{4}=0$, we get the following solutions:

$$
G(\xi, \tau)=\frac{\xi}{2 \sqrt{\pi}} \int_{0}^{\tau} \frac{(\tau-s)}{s \sqrt{s}} \exp \left(\frac{-\xi^{2}}{4 s}-\left(2 i \omega+M^{2}+\frac{1}{K}\right) s\right) \mathrm{d} s
$$

respectively

$$
G(\xi, \tau)=\frac{\xi}{2 \sqrt{\pi}} \int_{0}^{\tau} \frac{(\tau-s)^{2}}{s \sqrt{s}} \exp \left(\frac{-\xi^{2}}{4 s}-\left(2 i \omega+M^{2}+\frac{1}{K}\right) s\right) \mathrm{d} s
$$

for a Newtonian fluid performing the same motion.

\section{Graphical results and discussion}

Unsteady MHD accelerated flows of generalized Burgers? fluid over a rigid plate embedded in a porous medium have been examined. The governing partial differential equations for velocity have been analytically solved by the Laplace transform technique. In order to understand the nature of the flow and its dependence on the involved parameters $(M, K$, and $\omega$ ), the graphical results for dimensionless velocity $G$ versus $\xi$ and $\tau$ are plotted. We note that Figures 1-5 have been sketched for the constant accelerated flow. However, it is observed that the variations of parameters in variable accelerated flow are found qualitatively similar to the effects plotted for constant accelerated flow. Therefore, the graphical results for variable accelerated flow are not included. Further, each figure (a) and (b) depicts the behavior of real and imaginary parts of velocity. In all these figures, the same values of $\alpha=0.1, \beta=0.2, \gamma=0.3, \eta=0.4$ are used.

Figure 1 shows the graph of the velocity $G(\xi, \tau)$ for different values of magnetic parameter $M$. The range of magnetic field is chosen from 0 to 15 . It is noticed that in the absence of magnetic field velocity is maximum and then, by increasing the magnetic parameter $M$, the magnitude of both real and imaginary parts of velocity is decreasing. Such an effect may also be expected because of the fact that the transverse magnetic field produces a resistance force (Lorentz force) similar to drag force which tends to resist the flow thus reducing its velocity. Increasing the permeability parameter $K$ causes an increase in the magnitude of velocity, which is quite opposite to that of $M$ as shown in Figure 2. This is in accordance with the fact that an increase in permeability of the porous medium reduces the drag force. Consequently, this causes the velocity to increase. The behavior of rotation parameter $\omega$ is shown in Figure 3. It is noticed that the magnitude of the real part of velocity is a decreasing function of $\omega$. However, the imaginary part explains that velocity first increases in the range of $\xi=0$ to 0.5 and then, for the further large values of $\xi$, the velocity decreases when $\omega$ is increased and finally approaches zero. The variation of velocity along time $\tau$ for different values of $K$ and $\xi$ is shown in Figure 4. It is observed from Figure 4(a) that initially, when time is zero, the velocity is zero and the fluid is at rest. This is also true in view of the initial condition given by Eq. (7) $)_{1}$. Further, as time advances, the velocity of the fluid increases. However, for large values of $\xi$, the velocity is decreasing and ultimately approaching zero when $\xi$ tends to infinity. Physically, this is true due to the fact that faraway from the plate, there is no disturbance. From Figure 4(b), it is found that the variation in the imaginary part of velocity along time $\tau$ for different values of $\xi$ and $M$ is opposite when compared with the real part of velocity. It is further noted that at $\xi=0$, for $\tau>0$, the fluid is at rest. This is also true because of the fact that at $\xi=0$, the imaginary 


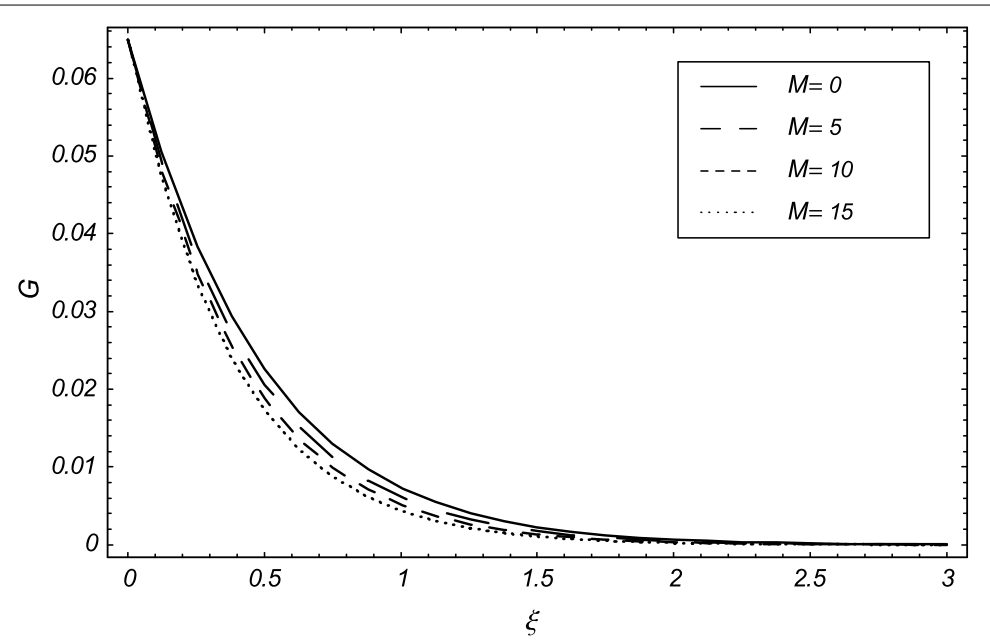

(a)

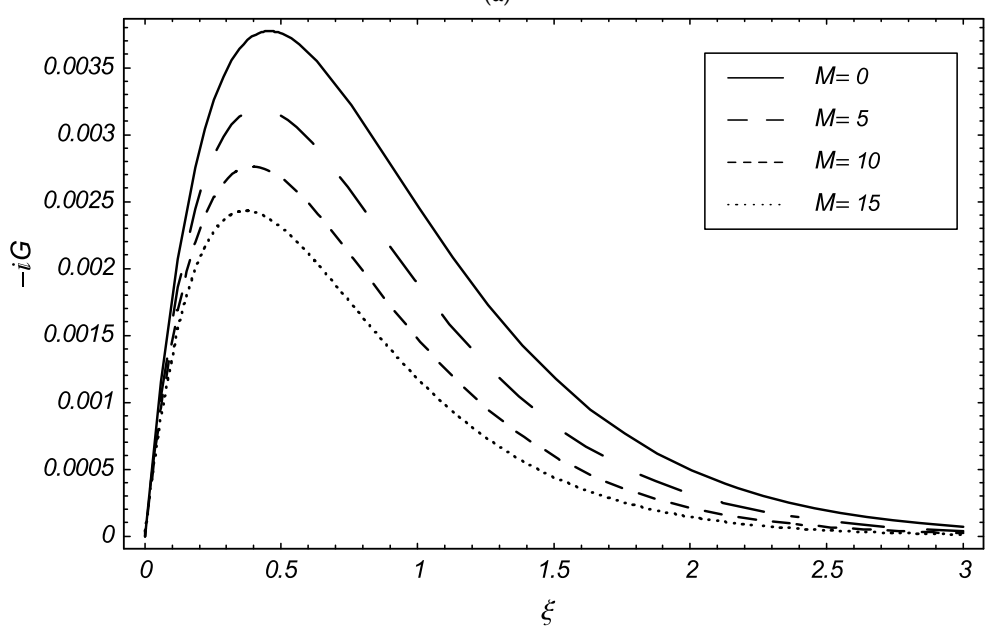

(b)

Figure 1 Velocity profiles for different values of $M$ when $\omega=2, K=0.5, \tau=0.068$.

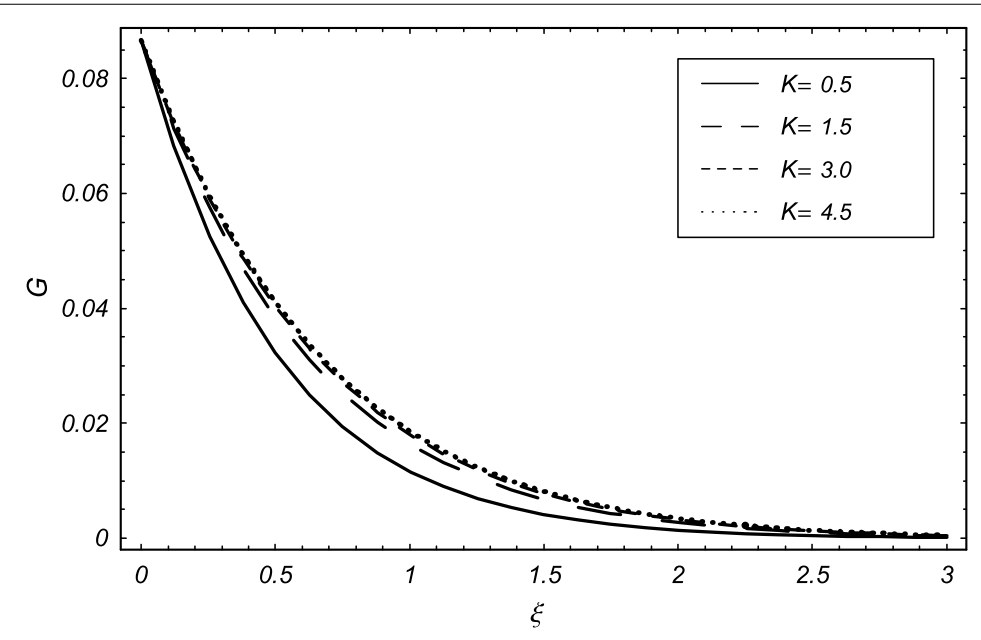

(a)

Figure 2 Velocity profiles for different values of $K$ when $\omega=2, M=0.5, \tau=0.089$. 


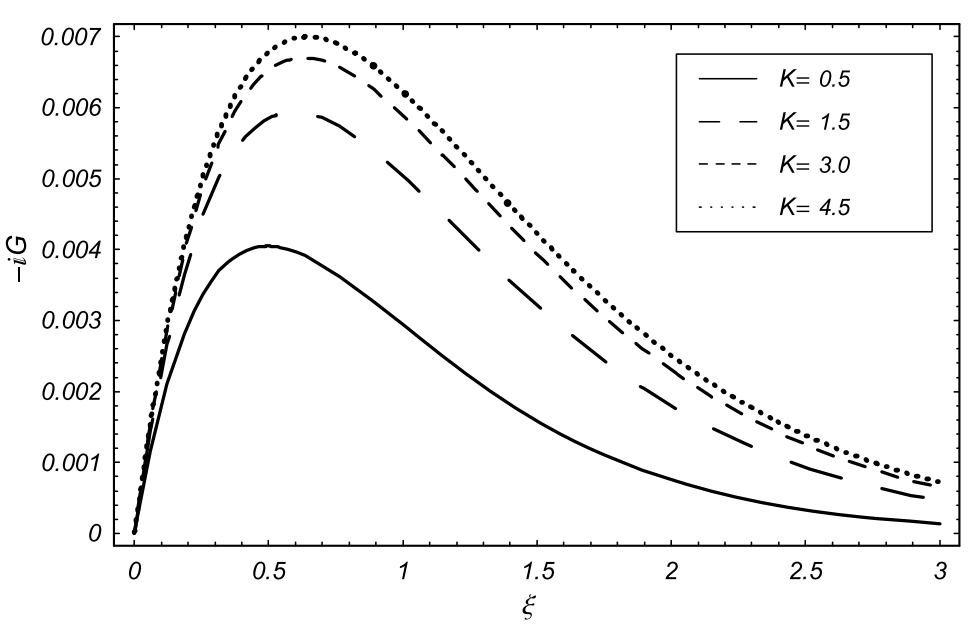

(b)

Figure 2 Continued

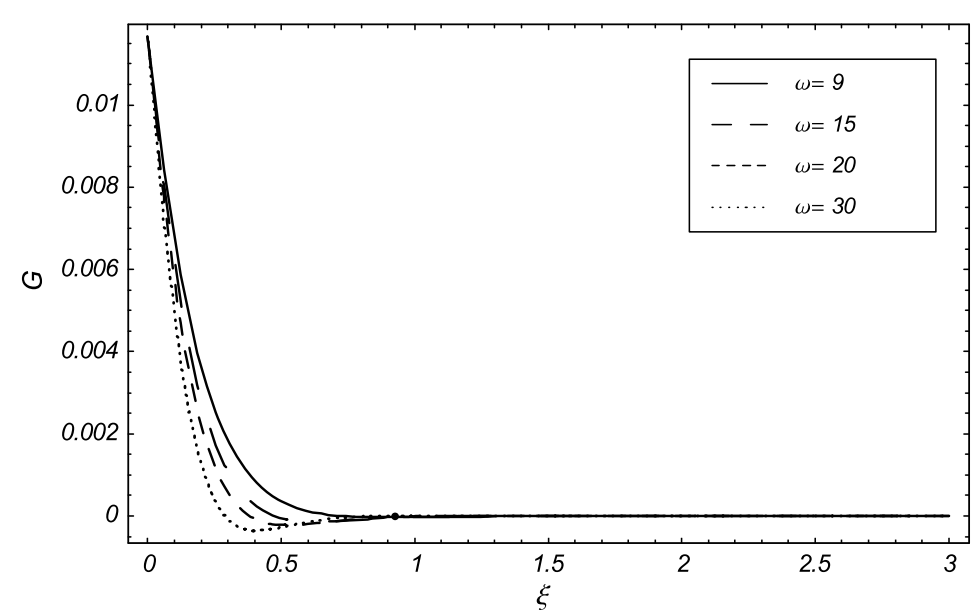

(a)

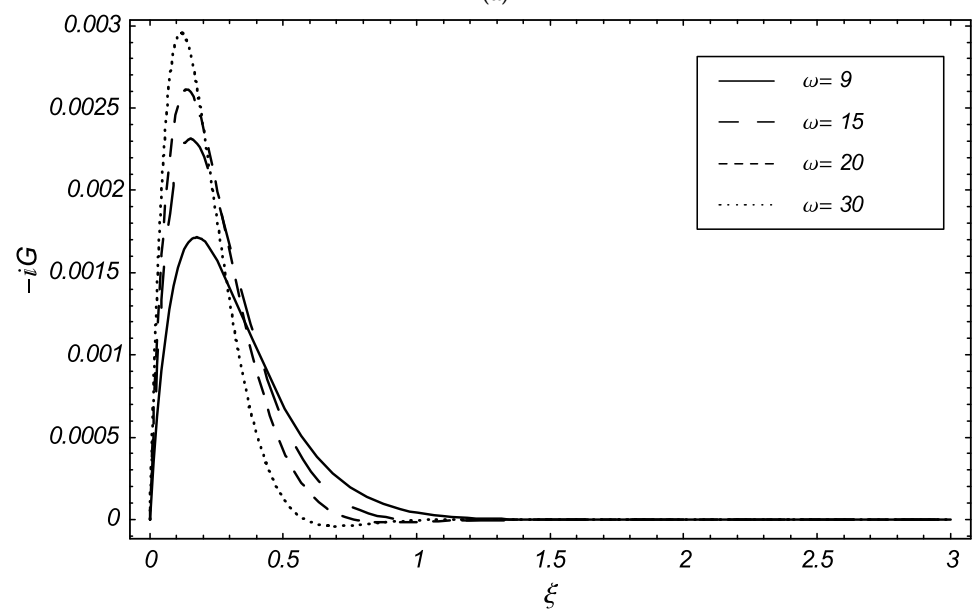

(b)

Figure 3 Velocity profiles for different values of $\omega$ when $M=2, K=0.5, \tau=0.018$. 


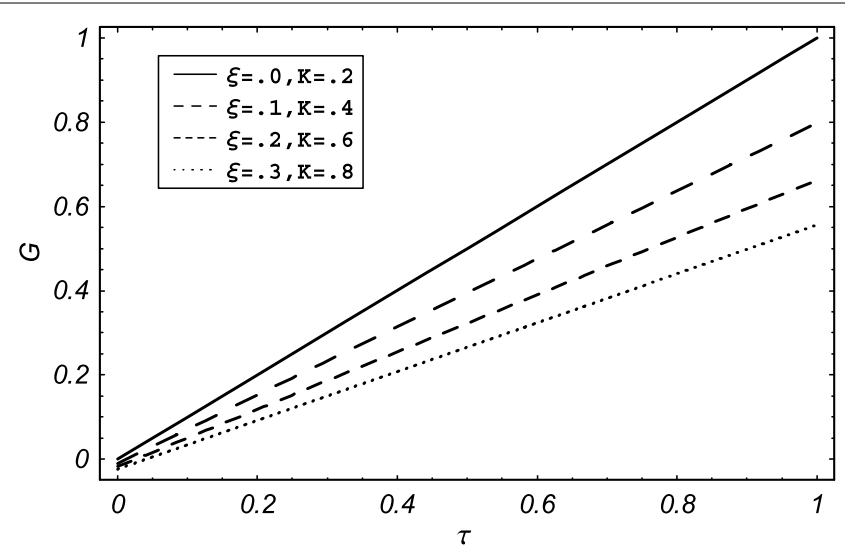

(a)

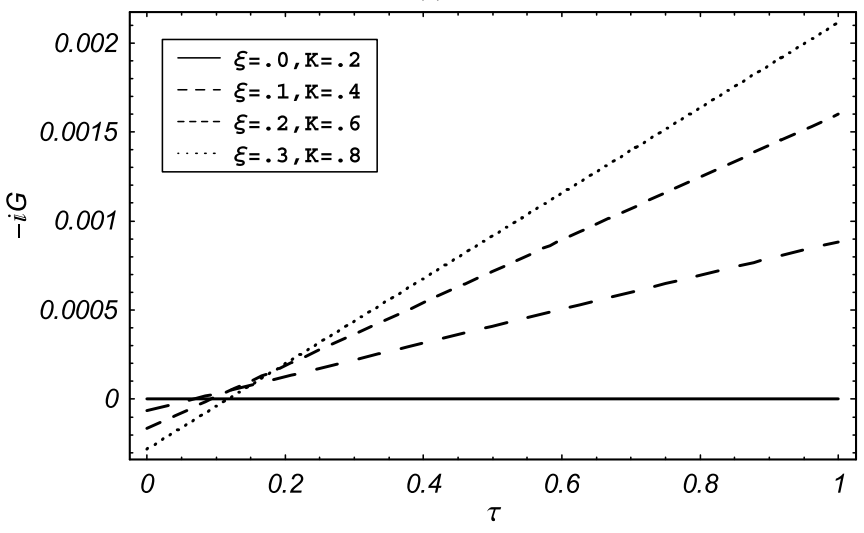

(b)

Figure 4 Variation of velocity along time for different values of $K$ and $\xi$ when $\omega=0.2, M=2$.

part of velocity is zero as we can see from boundary condition (6) $)_{1}$. The variation of velocity along $\tau$ for different values $\xi$ and $M$ is studied in Figure 5 . It is found that the behavior of velocity is quite identical with that of Figure 4 .

\section{Concluding remarks}

The objective of the present work was to determine the exact solutions for the accelerated flows in a rotating frame. The electrically conducting generalized Burgers? fluid is passing through a porous medium. Solutions for velocity have been obtained using the Laplace transform. Influences of emerging parameters on velocity have been shown in several graphs and the following observations have been noted.

- Under the MHD influence, fluid velocity decreases with an increase in the magnetic parameter $M$.

- The permeability parameter $K$ of the porosity yields an opposite effect to that of $M$.

- The rotation parameter $\omega$ decreases the velocity magnitude for the real part while increases the imaginary part first and then decreases.

- The salient features of embedded parameters in constant and variable accelerated cases are similar in qualitative sense.

- In the case of second grade fluid, our results are identical with those of Khan et al. [22]. 


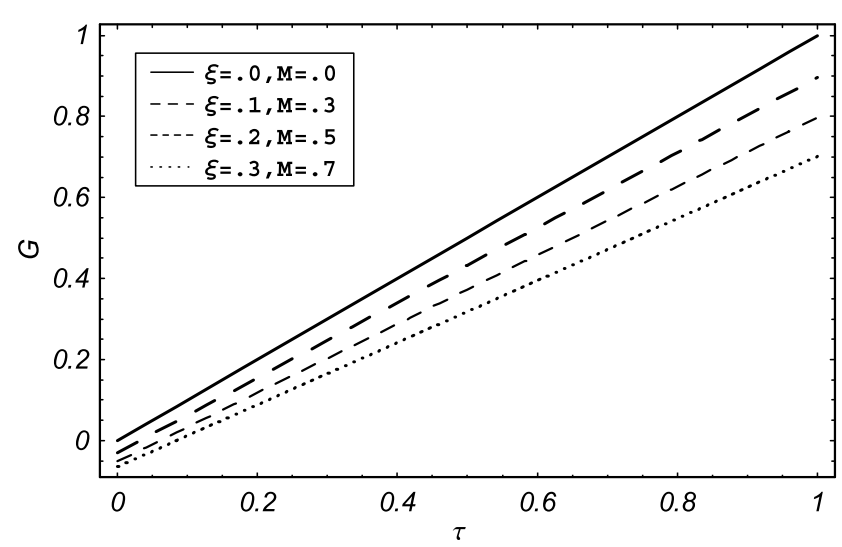

(a)

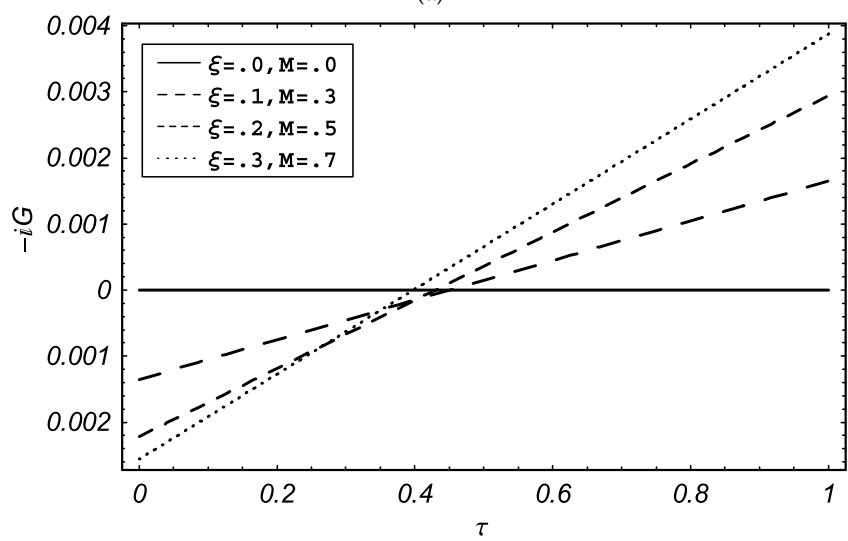

(b)

Figure 5 Variation of velocity along time for different values of $M$ and $\xi$ when $\omega=0.2, K=0.2$.

\section{Competing interests}

The authors declare that they have no competing interests.

\section{Authors? contributions}

All authors contributed equally to the writing of this paper. All authors read and approved the final manuscript.

\section{Author details}

'Department of Basic Sciences, College of Engineering, Majmaah University, P.O. Box 66, Majmaah, 11952, Saudi Arabia. ${ }^{2}$ Department of Mathematics, City University of Science and Information Technology, Peshawar, Pakistan. ${ }^{3}$ UTM Centre for Industrial \& Applied Mathematics (UTM-CIAM), Universiti Teknologi Malaysia, UTM Skudai, Johor Bahru, 81310,

Malaysia. ${ }^{4}$ Department of Mathematical Sciences, Faculty of Science, Universiti Teknologi Malaysia, UTM Skudai, Johor Bahru, 81310, Malaysia.

\section{Acknowledgements}

The authors would like to acknowledge the financial support of Majmaah University Saudi Arabia, the Ministry of Education Malaysia (MOE) and the Research Management Centre-UTM through vote numbers 06H67, 4F255 and 01621 for this research.

Received: 2 September 2014 Accepted: 1 December 2014 Published online: 15 January 2015

\section{References}

1. Krishnan, JM, Rajagopal, KR: A thermodynamic framework for the constitutive modeling of asphalt concrete: theory and application. J. Mater. Civ. Eng. 16, 155-166 (2004)

2. Ravindran, P, Krishnan, JM, Rajagopal, KR: A note on the flow of a Burgers? fluid in an orthogonal rheometer. Int. J. Eng. Sci. 42, 1973-1985 (2004)

3. Vieru, D, Fetecau, C, Fetecau, C: Flow of a viscoelastic fluid with the fractional Maxwell model between two side walls perpendicular to a plate. Appl. Math. Comput. 200, 459-464 (2008)

4. Vieru, D, Fetecau, C, Fetecau, C: Flow of a generalized Oldroyd-B fluid due to a constantly accelerating plate. Appl. Math. Comput. 201, 834-842 (2008) 
5. Athar, M, Fetecau, C: Unsteady flow of a generalized Maxwell fluid with fractional derivative due to a constantly accelerating plate. Comput. Math. Appl. 57, 596-603 (2009)

6. Nazar, M, Fetecau, C, Vieru, D, Fetecau, C: New exact solutions corresponding to the second problem of Stokes? for second grade fluids. Nonlinear Anal., Real World Appl. 11, 584-591 (2010)

7. Griffiths, PT, Garrett, SJ, Stephen, SO: The neutral curve for stationary disturbances in rotating disk flow for power-law fluids. J. Non-Newton. Fluid Mech. 213, 73-81 (2014)

8. Xie, ZY, Jian, YJ: Rotating electroosmotic flow of power-law fluids at high zeta potentials. Colloids Surf. A, Physicochem. Eng. Asp. 461, 231-239 (2014)

9. Deka, RK, Paul, A: Flow past an impulsively started horizontal cylinder in a rotating fluid. Int. J. Fluid Mech. Res. 40, 459-467 (2013)

10. Deka, RK, Paul, A, Chaliha, A: Transient free convection flow past an accelerated vertical cylinder in a rotating fluid. Ain Shams Eng. J. 5, 505-513 (2014)

11. Fetecau, C, Hayat, T, Khan, M, Fetecau, C: A note on longitudinal oscillations of a generalized Burgers fluid in cylindrical domains. J. Non-Newton. Fluid Mech. 165, 350-361 (2010)

12. Jamil, M, Fetecau, C: Some exact solutions for rotating flows of a generalized Burgers? fluid in cylindrical domain J. Non-Newton. Fluid Mech. 165, 23-24 (2010)

13. Siddiqui, AM, Rana, MA, Ahmed, N: Magnetohydrodynamics flow of a Burgers? fluid in an orthogonal rheometer. Appl. Math. Model. 34, 2881-2892 (2010)

14. Khan, M, Ali, SH, Fetecau, C: Exact solutions of accelerated flows for a Burgers? fluid. I. The case $\gamma<\lambda^{2} / 4$. Appl. Math. Comput. 203, 881-894 (2008)

15. Shahzad, F, Hayat, T, Ayub, M: Stokes? first problem for the rotating flow of a third grade fluid. Nonlinear Anal., Real World Appl. 9, 1794-1799 (2008)

16. Hayat, T, Khan, SB, Khan, M: Exact solution for rotating flows of a generalized Burgers? fluid in a porous space. Appl. Math. Model. 32, 749-760 (2008)

17. Hayat, T, Fetecau, C, Sajid, M: Analytical solution for MHD transient rotating flow of a second grade fluid in a porous space. Nonlinear Anal., Real World Appl. 9, 1619-1627 (2008)

18. Hayat, T, Fetecau, C, Sajid, M: On MHD transient flow of a Maxwell fluid in a porous medium and rotating frame. Phys. Lett. A 372, 1639-1644 (2008)

19. Hayat, T, Iram, S, Javeed, T, Asghar, S: Shrinking flow of second grade fluid in a rotating frame: an analytical solution. Commun. Nonlinear Sci. Numer. Simul. 15, 2932-2941 (2010)

20. Siddiqui, AM, Rana, MA, Ahmed, N: Effects of hall current and heat transfer on MHD flow of a Burgers? fluid due to a pull of eccentric rotating disks. Commun. Nonlinear Sci. Numer. Simul. 13, 1554-1570 (2008)

21. Abelman, S, Momoniat, E, Hayat, T: Couette flow of a third grade fluid with rotating frame and slip condition. Nonlinear Anal., Real World Appl. 10, 3329-3334 (2009)

22. Khan, I, Farhad, A, Sharidan, S, Norzieha, M: Exact solutions for accelerated flows of a rotating second grade fluid in a porous medium. World Appl. Sci. J. 9, 55-68 (2010) (special issue of applied math)

23. Roberts, GE, Kaufman, H: Table of Laplace Transforms. Saunders, Philadelphia (1968)

\section{Submit your manuscript to a SpringerOpen ${ }^{\circ}$ journal and benefit from:}

- Convenient online submission

Rigorous peer review

- Immediate publication on acceptance

- Open access: articles freely available online

- High visibility within the field

- Retaining the copyright to your article 